\section{From the Desk of Editor in Chief}

Alhamdulillah. By the grace of God AKMMC Journal volume 7 Number 2 July 2016 issue is published in time. I am grateful to the authors. coauthors, reviewers, executive editor, assistant editors, member of editorial and advisory board for their help \& support and some pharmaceticuals, clinics for giving us advertisement. Like previous other issue present issue will also be enjoyable to the readers, for the quality of the original, review article and interesting case reports. Editorial on Good Medical Practice (Duties of a Registered Doctor) highlighted the issues of good clinical care, maintaining good medical practical practice, relationship with patients, teaching \& training, working with collegues, probity and health. This is very important in formulating own guideline on good medical practice for registered doctors in Bangladesh. Original article on "Ante-natal care practices in some selected rural areas of Bangladesh" a cross sectional study on ante-natal care practices in some selected rural areas of Bangladesh, concluded that education of mothers is an important and determinant of ante-natal visit. Second original article on" Presence of Lewis blood group among Bangladeshi population," emphasised the need of detecting Lewis antibody in patients with multiple transfusion on having atypical or irregular antibody should be regularly tested for presence of Lewis antibody which may cause irregular agglutination interfering with compatibility testing. 3rd original article on "Post surgical outcome of dorsolateral Onlay Urethroplasty" found that it is a technically fixable, easily adaptable and successful procedure for standard management of urethral stricurte involving the anterior urethra. $4^{\text {th }}$ original article on "Antinociceptive and anti-inflammatory effects of combined administration of $\alpha$-tocopherol and morphine in acetic acid induced writhing test" concluded that combined administration of morphine sulphate, and $\alpha$-tocopherol were more effective in lowering the nocceptive and inflammatory pain than individual administration of morphine. $5^{\text {th }}$ "Original article on study of Rhesus genotype and phenotype in Bangladeshi population attended in a tertiary care hospital transfusion medicine department "findings may help to formulate strategy to prevent $\mathrm{Rh}$ sensitization, may thus also be useful in disputed paternity and genetic study in Bangladesh. Review article on "Zika virus-A global threat" highlighted AKMMC J 2016; 7(2) : 63-63 the important mosquite borne virus and its importance to prevent mother giving born to microcephally. Preventive measure like human to human blood transfusion, sativa, urine, sexual conteact and mother to fetus is a matter of great concern. Review article on "Prostate cancer" highlighted some of the important recent update on genetic factors, high fatty diet, hormone, inflammatory cytokines, trace element of zinc in the genesis of prostatic cancer. Case report on "Post chicken pox sequel in children" in three district population" highlighted the rare complication of chicken pox like neurological complication, hemophilia and osteomyelitis. So physician should keep in mind these post chicken pox sequel in children for better management and treatment. Another case report on "Complete androgen insensitivity syndrome a rare and late presentation with bilateral groin swelling in an 18 years old girl" describe a disease that causes resistance to androgen action. It is genetically $\mathrm{x}$-linked disorder with mutation of androgen receptor gene and can present with morphogenesis and differentiation of different structure especially external genitalia. Case report on "Chronic arsenic poisoning with skin cancer" described detremental effect of chronic arsenic exposure including skin lesion and two cases of histologically diagnosed skin cancer. As Bangladesh is a arsenic prevalent country preventive measure is advocated to prevent this complication. Last case report on "Rhinocerebral. Mucormycosis with post surgical complication in diabetes" described a rare fungal infection caused by Rhinocerebral mucormycosis in a diabetic ketoacidosis patient. The physician should be aware of the rare complication because of its rapid progression and high morality where early recognition and aggressive treatment is needed for increase survival.

Hopefully this issue will be interesting to be readers as other previous issues.

\section{Prof. Dr. Md. Tahminur Rahman}

Editor in Chief

AKMMC Journal

Email: mtahminur@yahoo.com

Phone: 008801817561519 